\title{
AN UNPUBLISHED THEOREM OF KRONECKER RESPECTING NUMERICAL EQUATIONS.
}

PRESIDENTIAL ADDRESS DELIVERED BEFORE THE AMERICAN MATHEMATICAL SOCIETY, DECEMBER 30, 1918.

BY PRESIDENT H. B. FINE.

1. IN the winter semester of 1890-91, when he lectured on algebra for the last time, Kronecker developed a theory of the algebraic equation with numerical coefficients to which he attached a great deal of importance but which unhappily he did not live to publish. Professor Hensel, of Marburg, Kronecker's literary legatee and editor of his works, possesses notes of these lectures which he kindly placed in my hands last summer with the very generous permission to make any use of them I might wish. In reading these notes, I came upon the theory to which I have referred, and it seemed to me so characteristic of Kronecker and so full of interest for the algebraist that $I$ have chosen it for the subject of this address.

All who have read Kronecker's later writings are familiar with his contention that the theory of the algebraic equation in its final form must be based solely on the rational integer, algebraic numbers being excluded and only such relations and operations being admitted as can be expressed in finite terms by means of rational numbers and therefore ultimately by means of integers. These lectures of 1890-91 are chiefly concerned with the development of such a theory, and in particular with the proof of two theorems which therein take the place of the fundamental theorem of algebra as commonly stated.

The first of these theorems relates to equations whose coefficients are rational functions of one or more variables and is characterised by Kronecker as the fundamental theorem of algebra in the algebraic sense. It is:

For every given algebraic equation

$$
F(x)=x^{n}-c_{1} x^{n-1}+\cdots+(-1)^{n} c_{n}=0
$$

there exists a congruence of the form

$$
F(x) \equiv\left(x-x_{1}\right)\left(x-x_{2}\right) \cdots\left(x-x_{n}\right) \quad\left(\operatorname{modd} f_{i}-c_{i}, g-c\right),
$$


where the $x_{i}$ 's are variables, the $f_{i}$ 's are the elementary symmetric functions of the $x_{i}$ 's, $c$ belongs to the domain of rationality of the coefficients $c_{i}$, and $g$ is a rational, integral, but in general nonsymmetric function of the $x_{i}$ 's whose form depends on the algebraic character of $F(x)$, and is such that the modular system whose elements are the $f_{i}-c_{i}$ and $g-c$ is prime.

Here, as always in Kronecker's algebraic writings, "exists" means "can be actually found by a limited number of rational operations." I shall not attempt to sketch the proof of this theorem since that would necessitate giving an account of a large part of Kronecker's arithmetical theory of algebraic functions. Moreover the theorem is fully discussed in the paper entitled "Ein Fundamentalsatz der allgemeinen Arithmetik" (Journal für die reine und angewandte Mathematik, volume 100). I have stated it mainly to give some indication of the methodthe use of indeterminates, such as the $x_{i}$ 's, and modular systems-by which Kronecker makes the introduction of the algebraic numbers unnecessary when the isolating of conjugate numbers is not required. That it is unnecessary in the latter case also Kronecker shows in his paper "Ueber den Zahlbegriff" (Journal für die reine und angewandte Mathematik, volume 101) and in the demonstration which follows.

Kronecker's second theorem, "the fundamental theorem of algebra in the arithmetical sense," relates to equations with rational numerical coefficients. It is:

Every rational integral function $f(x)$ of the nth degree with rational numerical coefficients can, by a variation of the coefficients which is definite in character but as small as one may please, be reduced to a product of $\nu$ linear and $(n-\nu) / 2$ quadratic factors, all with rational coefficients, $\nu$ being an integer determined by the coefficients of $f(x)$.

As hardly need be said, to establish this theorem in a manner meeting the requirements of the theory of which it forms a part, one must, without going beyond the domain of the rational numbers and therefore without at any point assuming the existence of linear factors of $f(x)$ or of roots of $f(x)=0$, derive a general method by which in the case of any given function the requisite variation of the coefficients may be obtained directly from the coefficients themselves. I shall give Kronecker's proof modified in certain particulars for convenience of exposition.

2. Since it is evidently only necessary to prove the theorem 
for the case in which $f(x)$ has no multiple factors or linear factors with rational coefficients, we shall suppose $f(x)$ to be without such factors. Then $f(x)=0$ has no rational roots, and therefore, from Kronecker's standpoint, no roots. But it may be the case that, $\delta$ representing a positive number taken as small as we please, there exist one or more $x$-intervals $\left(x^{\prime}, x^{\prime \prime}\right)$ such that

$$
f\left(x^{\prime}\right) f\left(x^{\prime \prime}\right)<0 \text { and }|f(x)|<\delta \text { for } x^{\prime} \leqq x \leqq x^{\prime \prime} .
$$

Any such interval we shall call a zero interval of $f(x)$, or, when convenient, a "root" of $f(x)=0$.

Every interval $(a, b)$ such that $f(a) f(b)<0$ contains at least one zero interval of $f(x)$.

For if $a<x<x+h<b$, and $g$ denote any number greater than all the values of $\left|f^{(r)}(x) / r !\right|(r=1,2, \cdots, n)$ in $(a, b)$, we shall have $|f(x+h)-f(x)|<\delta$ when $h<\delta /|g+\delta|$. Find an integer $\nu$ such that $(b-a) / \nu<\delta /(g+\delta)$, and divide $(a, b)$ into $\nu$ equal sub-intervals. Among these, since $f(a) f(b)<0$, there will be at least one, $\left(a_{v}, b_{v}\right)$, such that $f\left(a_{v}\right) f\left(b_{v}\right)<0$. Moreover if $x$ denote any number in $\left(a_{\nu}, b_{v}\right)$, both $\mid f(x)-$ $f\left(a_{\nu}\right) \mid<\delta$ and $\left|f\left(b_{\nu}\right)-f(x)\right|<\delta$, and either $f\left(a_{\nu}\right) f(x)<0$ or $f(x) f\left(b_{\nu}\right)<0$, and therefore $|f(x)|<\delta$. Hence $\left(a_{\nu}, b_{\nu}\right)$ is a zero interval of $f(x)$.

If throughout an interval $(a, b),\left|f^{\prime}(x)\right|>c$, where $c$ denotes some positive number, and if $\xi$ denote any number in $(a, b)$, then

$$
\operatorname{sgn} f^{\prime}(\xi)=\operatorname{sgn}[f(b)-f(a)] .
$$

That the sign of $f^{\prime}(x)$ is constant in $(a, b)$ follows from the preceding theorem. And if $a<x<x+h<b$, and $g$ have the same meaning as above, then since $f(x+h)-f(x)=$ $h\left[f^{\prime}(x)+h f^{\prime \prime}(x) / 2 !+\cdots\right]$, we shall have $\operatorname{sgn}[f(x+h)-f(x)]$ $=\operatorname{sgn} f^{\prime}(x)$ when $\left|h f^{\prime \prime}(x) / 2 !+\cdots\right|<\left|f^{\prime}(x)\right|$ and therefore when $h<c /(c+g)$. Hence, if $(a, b)$ be divided into intervals $\left(x_{i}, x_{i+1}\right)$ of the magnitude $c /(c+g)$ or less, we shall have $\operatorname{sgn}\left[f\left(x_{i+1}\right)-f\left(x_{i}\right)\right]=\operatorname{sgn} f^{\prime}(\xi)$ for every value of $i$ and therefore $\operatorname{sgn}[f(b)-f(a)]=\operatorname{sgn} f^{\prime}(\xi)$.

From this theorem it immediately follows that if $\left|f^{\prime}(x)\right|>c$ throughout $(a, b),(a, b)$ will contain a sub-interval $\left(x^{\prime}, x^{\prime \prime}\right)$ in which $|f(x)|<\delta$ only when $f(a) f(b)<0$, and then but one such sub-interval. It also follows that:

Between two consecutive zero intervals of $f(x),\left(x_{1}{ }^{\prime}, x_{1}{ }^{\prime \prime}\right)$ and 
$\left(x_{2}{ }^{\prime}, x_{2}{ }^{\prime \prime}\right)$, in both of which $\left|f^{\prime}(x)\right|>c$, there is a zero interval of $f^{\prime}(x)$.

For $\operatorname{sgn} f^{\prime}\left(x_{1}^{\prime \prime}\right)=\operatorname{sgn}\left[f\left(x_{1}^{\prime \prime}\right)-f\left(x_{1}^{\prime}\right)\right]=\operatorname{sgn} f\left(x_{1}^{\prime \prime}\right)=\operatorname{sgn} f\left(x_{2}^{\prime}\right)$ $=-\operatorname{sgn}\left[f\left(x_{2}{ }^{\prime \prime}\right)-f\left(x_{2}^{\prime}\right)\right]=-\operatorname{sgn} f^{\prime}\left(x_{2}{ }^{\prime}\right)$.

It is therefore evident that we shall be able to isolate the zero intervals of $f(x)$ if we can determine the size of an interval in which there cannot be both a zero interval of $f(x)$ and one of $f^{\prime}(x)$. As will be shown, this can be found from the identity $D=P(x) f(x)+Q(x) f^{\prime}(x)$ connecting $f(x), f^{\prime}(x)$, and the discriminant $D$ of $f(x)$. We therefore proceed to establish this identity by a method which does not assume the existence of linear factors of an integral function.

3. The necessary and sufficient condition that the functions

and

$$
F(x)=\prod_{i=1}^{m}\left(x-x_{i}\right)=x^{m}+\sum_{r=1}^{m}(-1)^{r} f_{r} x^{m-r}
$$

$$
G(x)=\prod_{k=1}^{n}\left(x-y_{k}\right)=x^{n}+\sum_{r=1}^{n}(-1)^{r} g_{r} x^{n-r}
$$

have a common factor is the vanishing of the rational integral function of the coefficients $f_{r}, g_{r}$, defined by

$$
\begin{aligned}
\operatorname{Res}(F, G)=\prod_{i k}\left(x_{i}-y_{k}\right)= & \prod_{i=1}^{m} G\left(x_{i}\right) \\
& =(-1)^{m n} \prod_{k=1}^{n} F\left(y_{k}\right)=R\left(f_{r}, g_{r}\right) .
\end{aligned}
$$

The roots of the equation $F(x) G(x)=0$ being $x_{1}, \cdots, x_{m}$, $y_{1}, \cdots, y_{n}$, and the expressions for $R\left(f_{r}, g_{r}\right)$ in terms of these roots being $\prod_{i=}^{m} G\left(x_{i}\right),(-1)^{m n} \prod_{k=1}^{n} F\left(y_{k}\right)$, we have, by the interpolation formula of Lagrange,

$$
\begin{aligned}
& R\left(f_{r}, g_{r}\right)=G(x) \sum_{i=1}^{m} \frac{F(x)}{x-x_{i}} \frac{\prod_{i=1}^{m} G\left(x_{i}\right)}{G\left(x_{i}\right) F^{\prime}\left(x_{i}\right)} \\
& \quad+(-1)^{m n} F(x) \sum_{k=1}^{n} \frac{G(x)}{x-y_{k}} \frac{\prod_{k=i}^{n} F\left(y_{k}\right)}{F\left(y_{k}\right) G^{\prime}\left(y_{k}\right)} .
\end{aligned}
$$

Evidently the coefficient of $G(x)$ in this identity can be reduced 
to the form $\sum_{i=1}^{m}\left[a_{0}\left(x_{i}\right) x^{m-1}+\cdots+a_{m-1}\left(x_{i}\right)\right] / F^{\prime}\left(x_{i}\right)$, where $a_{0}\left(x_{i}\right), \cdots, a_{m-1}\left(x_{i}\right)$ are integral functions of the $f_{r}, g_{r}$ and of $x_{i}$, of degree not higher than $m-1$ in $x_{i}$. But, by Euler's formulas, $\Sigma_{i=1}^{n} x_{i}{ }^{\lambda} / F^{\prime}\left(x_{i}\right)=1$ or 0 according as $\lambda=m-1$ or $<m-1$. Therefore the coefficient of $G(x)$ is a polynomial $\varphi(x)$, of degree $m-1$ at most, with coefficients which are integral functions of the $f_{r}, g_{r}$. The coefficient of $F(x)$ is reducible to a polynomial $\psi(x)$ of like character of degree $n-1$ at most. Therefore

$$
R\left(f_{r}, g_{r}\right)=\varphi(x) G(x)+\psi(x) F(x),
$$

where $\varphi(x)$ and $\psi(x)$ have the character just indicated.

Let

and

$$
F_{1}(x)=x^{m}+\sum_{r=1}^{m}(-1)^{r} c_{r} x^{m-r}
$$

$$
G_{1}(x)=x^{n}+\sum_{r=1}^{n}(-1)^{r} d_{r} x^{n-r}
$$

be functions with any given rational coefficients and which therefore are in general not resolvable into linear factors. We are to prove that the vanishing of $R\left(c_{r}, d_{r}\right)$ is the necessary and sufficient condition that $F_{1}(x)$ and $G_{1}(x)$ have a common factor. form

If in (1) we set $f_{r}=c_{r}, g_{r}=d_{r}$, we obtain an identity of the

$$
R\left(c_{r}, d_{r}\right)=\varphi_{1}(x) G_{1}(x)+\psi_{1}(x) F_{1}(x),
$$

from which it immediately follows that, if $F_{1}(x)$ and $G_{1}(x)$ have a common factor, $R\left(c_{r}, d_{r}\right)$ must be 0 . And we can show as follows that if $F_{1}(x)$ and $G_{1}(x)$ have no common factor, $R\left(c_{r}, d_{r}\right)$ cannot be 0 .

For if $F_{1}(x)$ and $G_{1}(x)$ have no common factor, we can by means of the euclidean algorithm derive an identity of the form $1=P_{1}(x) F_{1}(x)+Q_{1}(x) G_{1}(x)$, where $P_{1}(x)$ and $Q_{1}(x)$ are integral functions of $x$. This identity may be written

$$
\begin{aligned}
& 1=F(x) P_{1}(x)+G(x) Q_{1}(x)+\left(F_{1}(x)-F(x)\right) \\
& P_{1}(x)+ \\
&\left(G_{1}(x)-G(x)\right) Q_{1}(x),
\end{aligned}
$$

from which it follows that

$$
1 \equiv F(x) P_{1}(x)+G(x) Q_{1}(x) \quad\left(\operatorname{modd} f_{r}-c_{r}, g_{r}-d_{r}\right) .
$$


Setting $x=x_{1}, x_{2}, \cdots, x_{m}$, successively, and taking the product of the resulting congruences, we have

$1 \equiv \Pi G\left(x_{i}\right) \Pi Q_{1}\left(x_{i}\right) \equiv R\left(f_{r}, g_{r}\right) \cdot \Gamma\left(f_{r}\right) \quad\left(\operatorname{modd} f_{r}-c_{r}, g_{r}-d_{r}\right)$, where $\Gamma\left(f_{r}\right)$ is an integral function of the $f_{r}$ 's. Therefore finally, setting $f_{r}=c_{r}, g_{r}=d_{r}$, we have

$$
1=R\left(c_{r}, d_{r}\right) \cdot \Gamma\left(c_{r}\right),
$$

from which it follows, since $\Gamma\left(c_{r}\right)$ cannot be infinite, that $R\left(c_{r}, d_{r}\right)$ is not 0 .

In the following discussion, by the resultant of two functions of the form $f(x)=c_{0} x^{m}-c_{1} x^{m-1}+\cdots+(-1)^{m} c_{m}$ and $g(x)=d_{0} x^{n}-d_{1} x^{n-1}+\cdots+(-1)^{n} d_{n}$, we shall mean the number $R=c_{0}{ }^{n} d_{0}{ }^{m} R\left[c_{r} / c_{0}, d_{r} / d_{0}\right]$, and by the discriminant of $f(x)$ we shall mean $D=\operatorname{Res}\left(f, f^{\prime}\right) / c_{0}$. Evidently $R$ and $D$ are integral when the given coefficients are integral.

4. Let $f(x)=\Sigma_{i=0}^{n} a_{i} x^{n-i}$ and $\varphi(x)=\Sigma_{i=0}^{m} b_{i} x^{m-i}$ be two polynomials with integral coefficients and having no common factor, whose resultant $R$ is therefore an integer different from 0. By means of the identity $R=P(x) f(x)+Q(x) \varphi(x)$ we can determine an integer $s$ such that no interval of the magnitude $1 / s$ will contain zero intervals of both $f(x)$ and $\varphi(x)$.

For if $x_{1}, x_{2}$ denote any two values of $x$, we have identically

(1) $R=P\left(x_{1}\right) \frac{f\left(x_{2}\right)-f\left(x_{1}\right)}{x_{2}-x_{1}}\left(x_{1}-x_{2}\right)+P\left(x_{1}\right) f\left(x_{2}\right)+Q\left(x_{1}\right) \varphi\left(x_{1}\right)$.

Since $\left|a_{0} x^{n}\right|>\left|a_{1} x^{n-1}+\cdots\right|$ for $|x|>\left(\left|a_{0}\right|+a_{g}\right) /\left|a_{0}\right|$, where $a_{g}$ denotes the greatest of the numbers $\left|a_{i}\right|$, and similarly $\left|b_{0} x^{m}\right|>\left|b_{1} x^{m-1}+\cdots\right|$ for $|x|>\left(\left|b_{0}\right|+b_{g}\right) /\left|b_{0}\right|$, all the zero intervals of $f(x)$ and $\varphi(x)$ lie in the interval $(-c, c)$, where $c$ denotes the first integer greater than both the numbers $\left(\left|a_{0}\right|+a_{g}\right) /\left|a_{0}\right|$ and $\left(\left|b_{0}\right|+b_{g}\right) /\left|b_{0}\right|$.

Let $K$ denote the greatest of the three numbers derived from

$$
P(x) \frac{f(x+\sigma)-f(x)}{\sigma}=\psi(x, \sigma), \quad P(x), \text { and } Q(x)
$$

by replacing the coefficients by their absolute values, $x$ by $c$, and $\sigma$ by 1 . Then any integer $s$ such that

$$
K<(s-1)|R|
$$

will meet the requirement stated above. For if $s$ satisfy (2), 
and if also $-c<x_{1}, x_{2}<c$ and $\left|x_{1}-x_{2}\right|<1$, it will follow from (1), by dividing throughout by $(s-1)|R|$, that

$$
\frac{1}{s-1}<\left|x_{1}-x_{2}\right|+\left|f\left(x_{2}\right)\right|+\left|\varphi\left(x_{1}\right)\right|
$$

and therefore, if $\left|x_{1}-x_{2}\right|<1 / s$, that

$$
\left|f\left(x_{2}\right)\right|+\left|\varphi\left(x_{1}\right)\right|>\frac{1}{s(s-1)} .
$$

But since $x_{1}, x_{2}$ are independent variables subject only to the conditions $-c<x_{1}, x_{2}<c$ and $\left|x_{1}-x_{2}\right|<1 / s$, it follows from (3) that no interval of the magnitude $1 / s$ can contain zero intervals of both $f(x)$ and $\varphi(x)$. On the contrary, if it contains a zero interval of one of the functions, the other will remain numerically greater than $1 / s(s-1)$ throughout the interval.

We are now in a position to isolate and count the zero intervals of any polynomial $f(x)$. For if in the preceding discussion we suppose $\varphi(x)=f^{\prime}(x)$ and, having determined $s$, divide the interval $(-c, c)$ into equal sub-intervals of the magnitude $1 / s$ it follows from $\$ 2$ that those only of these subintervals contain zero intervals of $f(x)$ at whose extremities $f(x)$ has opposite signs, and that each such sub-interval contains one and but one zero interval of $f(x)$. Hence the number of such sub-intervals is the number of the zero intervals of $f(x)$. And since sgn $f(-c)=\operatorname{sgn} f(c)$ or $\operatorname{sgn} f(-c)=-\operatorname{sgn} f(c)$ according as $n$, the degree of $f(x)$, is even or odd, the number $\nu$ of the zero intervals of $f(x)$ is even (or 0 ) or odd according as $n$ is even or odd.

5. We are now equipped for proving that part of Kronecker's theorem which relates to the linear factors. For to the conditions used in determining $s$ in $\S 4$ let us add, for the case in which $\varphi(x)=f^{\prime}(x)$ and therefore $R=D=\operatorname{Disc} f(x)$, the following:

$\left|\frac{f(x+\sigma)-f(x)}{\sigma}\right|<(s-1)|D|$, for $|x|<c$ and $|\sigma| \leqq 1$.

Then, for $x_{1}, x_{2}$ such that $-c<x_{1}, x_{2}<c$ and $\left|x_{2}-x_{1}\right|<1$, we have

$$
\left|f\left(x_{2}\right)-f\left(x_{1}\right)\right|<(s-1)|D| \cdot\left|x_{2}-x_{1}\right| .
$$

Divide $(-c, c)$ into equal intervals of the magnitude $1 / s$, and 
of these intervals let $\left(x^{\prime}, x^{\prime \prime}\right)$ be any one such that $f\left(x^{\prime}\right) f\left(x^{\prime \prime}\right)$ $<0$. Then, $r$ denoting an integer which may be taken as great as we please, divide $\left(x^{\prime}, x^{\prime \prime}\right)$ into $r|D|$ equal sub-intervals. Of these sub-intervals there will be one and but one at whose extremities $f(x)$ has opposite signs. If this sub-interval be $\left(\xi^{\prime}, \xi^{\prime \prime}\right)$, it follows from (1) that, if $\xi^{\prime} \leqq \xi \leqq \xi^{\prime \prime}$, then both $\left|f(\xi)-f\left(\xi^{\prime}\right)\right|<1 / r$ and $\left|f\left(\xi^{\prime \prime}\right)-f(\xi)\right|<1 / r$ and therefore, since either $f\left(\xi^{\prime}\right) f(\xi)<0$ or $f(\xi) f\left(\xi^{\prime \prime}\right)<0$, that $|f(\xi)|<1 / r$. Hence $\left(\xi^{\prime}, \xi^{\prime \prime}\right)$ is a zero interval of $f(x)$ characterized by the inequalities

(2) $f\left(\xi^{\prime}\right) f\left(\xi^{\prime \prime}\right)<0$ and $|f(\xi)|<1 / r$ for $\xi^{\prime} \leqq \xi \leqq \xi^{\prime \prime}$.

There are $\nu$ such sub-intervals $(\S 4)$. Represent them by

$$
\left(\xi_{1}{ }^{\prime}, \xi_{1}{ }^{\prime \prime}\right),\left(\xi_{2}{ }^{\prime}, \xi_{2}{ }^{\prime \prime}\right), \cdots\left(\xi_{\nu}{ }^{\prime}, \xi_{\nu}{ }^{\prime \prime}\right) \text {. }
$$

We are to prove that if $\xi_{i}$ denote any number in $\left(\xi_{i}{ }^{\prime}, \xi_{i}{ }^{\prime \prime}\right)$, we can reduce $f(x)$ to the form

(4) $f(x)=\left(x-\xi_{1}\right)\left(x-\xi_{2}\right) \cdots\left(x-\xi_{\nu}\right) Q(x)+R(x)$,

where $Q(x)$ is a polynomial without zero intervals and $R(x)$ a polynomial whose coefficients are as small as we please.

Represent $\Pi_{k=1}^{\nu}\left(x-\xi_{k}\right)$ by $\varphi(x)$. Then since $R\left(\xi_{k}\right)=f\left(\xi_{k}\right)$ for $k=1,2, \cdots, \nu$, we have, by the interpolation formula of Lagrange,

$$
R(x)=\sum_{k=1}^{\nu} \frac{f\left(\xi_{k}\right)}{\varphi^{\prime}\left(\xi_{k}\right)} \frac{\varphi(x)}{x-\xi_{k}} .
$$

But $\varphi^{\prime}\left(\xi_{k}\right)=\Pi\left(\xi_{k}-\xi_{i}\right)(i=1,2, \cdots, k-1, k+1, \cdots, \nu)$ and $\left|\xi_{k}-\xi_{i}\right|>1 / s$. For between the intervals $\left(\xi_{1}{ }^{\prime}, \xi_{1}{ }^{\prime \prime}\right)$ and $\left(\xi_{2}^{\prime}, \xi_{2}^{\prime \prime}\right)$, say, there lies at least one interval $\left(\eta^{\prime}, \eta^{\prime \prime}\right)$ of magnitude $1 / r s|D|$ which is a zero interval of $f^{\prime}(x)$, and if $\eta$ denote any number in $\left(\eta^{\prime}, \eta^{\prime \prime}\right)$, since no interval of the magnitude $1 / s$ can contain zero intervals of both $f(x)$ and $f^{\prime}(x)$, we have $\eta-\xi_{1} \geqq 1 / s-1 / r s|D|, \xi_{2}-\eta \geqq 1 / s-1 / r s|D|$ and therefore $\xi_{2}-\xi_{1} \geqq 2 / s-2 / r s|D|$. Hence $\xi_{2}-\xi_{1}>1 / s$ if, as we shall suppose, $r|D| \geqq 2$. Therefore

$$
\left|\frac{f\left(\xi_{k}\right)}{\varphi^{\prime}\left(\xi_{k}\right)}\right|<\frac{s^{\nu-1}}{r} \quad(k=1,2, \cdots, \nu) .
$$

Again $\varphi(x) /\left(x-\xi_{k}\right)$ is a polynomial in $x$, of degree $\nu-1$, whose coefficients after the first are less respectively than the numbers 
$(\nu-1) c, \quad(\nu-1)(\nu-2) c^{2} / 2, \cdots, c^{\nu-1}$. Therefore, if $k$ denote an integer greater than all these numbers, it follows from (5) that $R(x)$ can be reduced to the form

$$
R(x)=\frac{\nu s^{\nu-1} k}{r} S(x)=\frac{1}{r^{\prime}} S(x), \text { if } r=\nu s^{\nu-1} k r^{\prime},
$$

where the coefficients of $S(x)$ are all numerically less than 1 , and $r^{\prime}$ is an arbitrary integer which may be taken as great as we please, great enough, therefore, to make the coefficients of $R(x)$ as small as we please.

Moreover $r^{\prime}$ can be so taken that $Q(x)$ will have no zero interval. For $Q(x)$ will have no zero interval if $f(x)-R(x)$ has, like $f(x), \nu$ zero intervals. But consider the polynomial $f_{t}(x)=f(x)-t R(x)$, where $t$ is supposed to vary (through rational values) from 0 to 1 , and therefore $f_{t}(x)$ from $f(x)$ to $f(x)-R(x)$. It will be proved in the following section that the number of the zero intervals of a polynomial with variable coefficients changes only when the coefficients pass through values for which the discriminant of the polynomial vanishes (that is, becomes less than any assignable number). Hence, as $t$ varies from 0 to 1 , the number of the zero intervals of $f_{t}(x)$ is always $\nu$ unless Disc $f_{t}(x)$ vanishes. But Disc $f_{t}(x)$ can be reduced to the form $D+\psi$, where $\psi$ is an integral function of the coefficients of $f(x)$ and $t R(x)$, and therefore cannot vanish if $|\psi|<|D|$. But it will readily be seen that $|\psi|<|D|$ if, $g$ denoting the greatest coefficient in the development of $D\left[\left|a_{0}\right|, \cdots\left|a_{n-\nu}\right|,\left|a_{n-\nu+1}\right|+1 / r^{\prime}, \ldots,\left|a_{n}\right|+1 / r^{\prime}\right]$ in powers of $1 / r^{\prime}$, we take $r^{\prime}$ such that $1 / r^{\prime}<|D| /(|D|+g)$. Therefore if $r^{\prime}>(|D|+g) /|D|, Q(x)$ will have no zero interval.

6. But it remains to show that for a polynomial with variable coefficients the vanishing of the discriminant is the necessary condition for a change in the number of zero intervals. Kronecker establishes this theorem by aid of the notion of the characteristic of an equation or integral function.

Let $V(x)$ and $W(x)$ be two polynomials of the same degree with rational coefficients and such that $R(V, W), R\left(V, V^{\prime}\right)$, and $R\left(W, W^{\prime}\right)$ are all different from 0 ; and let

$$
\Delta(x)=\mid \begin{array}{cc}
V(x) & W(x) \\
V^{\prime}(x) & W^{\prime}(x)
\end{array} ! .
$$


By the method of $\S 4$ determine an integer $s$ such that no interval of the magnitude $1 / s$ contains two zero intervals of $\varphi(x)=V(x) W(x)$, and, representing the interval in which all the zero intervals of $\varphi(x)$ lie by $(-c, c)$, divide $(-c, c)$ into equal intervals $\left(x_{1}, x_{2}\right),\left(x_{2}, x_{3}\right), \cdots$ of the magnitude $1 / s$. Represent each of these intervals which is a zero interval of $V(x)$ by any number $\xi$ belonging to the interval, and let $\eta$ have the corresponding meaning for $W(x)$ and $\zeta$ for $\varphi(x)$. Finally let the symbol sgn $f(x)$ mean 1 or -1 according as $f(x)$ is positive or negative.

If $\left(x_{i}, x_{i+1}\right)$ be a zero interval of $\varphi(x)$, we have, by $\S 2$, $\operatorname{sgn} \varphi^{\prime}(\zeta)=\operatorname{sgn}\left[\varphi\left(x_{i+1}\right)-\varphi\left(x_{i}\right)\right]=\frac{1}{2}\left[\operatorname{sgn} \varphi\left(x_{i+1}\right)-\operatorname{sgn} \varphi\left(x_{i}\right)\right]$. But if $\left(x_{i}, x_{i+1}\right)$ be not a zero interval, $\operatorname{sgn} \varphi\left(x_{i+1}\right)-\operatorname{sgn} \varphi\left(x_{i}\right)$ $=0$. Therefore if $\Sigma_{\zeta}$ denote a sum extended over all the zero intervals of $\varphi(x)$, we have, since the degree of $\varphi(x)$ is even,

that is,

$$
\Sigma_{\zeta} \operatorname{sgn} \varphi^{\prime}(\zeta)=\frac{1}{2} \operatorname{sgn} \varphi(c)-\frac{1}{2} \operatorname{sgn} \varphi(-c)=0,
$$

$$
\Sigma_{\xi} \operatorname{sgn} V^{\prime}(\xi) W(\xi)+\Sigma_{\eta} \operatorname{sgn} V(\eta) W^{\prime}(\eta)=0,
$$

the sums $\Sigma_{\xi}$ and $\Sigma_{\eta}$ being extended over all the zero intervals of $V(x)$ and $W(x)$ respectively. And from (1) and (2) it at once follows that

$$
\Sigma_{\xi} \operatorname{sgn} \Delta(\xi)=\Sigma_{\eta} \operatorname{sgn} \Delta(\eta) .
$$

The integer or fraction $\mathbf{X}(V, W)$ defined by the equations

$$
\mathrm{X}(V, W)=-\frac{1}{2} \Sigma_{\xi} \operatorname{sgn} \Delta(\xi)=-\frac{1}{2} \Sigma_{\eta} \operatorname{sgn} \Delta(\eta)
$$

is called the characteristic of the functions $V(x)$ and $W(x)$. In particular

$$
\mathrm{X}\left(V, V+V^{\prime}\right)=\frac{1}{2} \Sigma_{\xi} \operatorname{sgn} V^{\prime 2}(\xi) .
$$

Therefore since $\operatorname{sgn} V^{\prime 2}(\xi)=1$, the number of the zero intervals of any polynomial $V(x)$ which has no multiple factors is $2 \mathrm{X}\left(V, V+V^{\prime}\right)$, or, more simply, $2 \mathrm{X}\left(V, V^{\prime}\right)$, if in defining $\mathbf{X}\left(V, V^{\prime}\right)$ we restrict ourselves to the first of the equations (4). It is convenient to call $\mathrm{X}\left(V, V^{\prime}\right)$ the characteristic of $V(x)$.

If $V(x)=\Sigma_{i=0}^{n} v_{i} x^{i}, W(x)=\Sigma_{i=0}^{n} w_{i} x^{i}$, and the coefficients $v_{i}$, $w_{i}$ are supposed to vary continuously (through rational values), the only possibilities of a change in the value of $\mathrm{X}(V, W)$ are such as present themselves when the $v_{i}, w_{i}$ pass through values for which $\Delta(V, W)=V(x) W^{\prime}(x)-V^{\prime}(x) W(x)$ vanishes (that is, becomes numerically less than every assignable 
positive number $\delta$ ) at the same time as either $V(x)$ or $W(x)$ that is, when (1) $V(x)=0$ and $W(x)=0$, or (2) $V(x)=0$ and $V^{\prime}(x)=0$, or $(3) W(x)=0$ and $W^{\prime}(x)=0$. But, for all values of the parameter $t$,

$\left|\begin{array}{l}V(x), W(x) \\ V^{\prime}(x), W^{\prime}(x)\end{array}\right|=\left|\begin{array}{l}V(x), W(x)-t V(x) \\ V^{\prime}(x), W^{\prime}(x)-t V^{\prime}(x)\end{array}\right|=\left|\begin{array}{l}V(x)-t W(x), W(x) \\ V^{\prime}(x)-t W^{\prime}(x), W^{\prime}(x)\end{array}\right|$

and therefore $\mathrm{X}(V, W)=\mathrm{X}(V, W-t V)=\mathrm{X}(V-t W, W)$, and the only one of the possible conditions for a change of value (1), (2), (3) which is common to these three expressions for $\mathbf{X}(V, W)$ is (1) $V(x)=0$ and $W(x)=0$; since for any values of $v_{i}, w_{i}$, and $x$ for which $V(x) W^{\prime}(x)-V^{\prime}(x) W(x)$ vanishes (except those for which both $V(x)$ and $W(x)$ vanish), $t$ may be so chosen that both $W(x)-t V(x)$ and $V(x)-t W(x)$ are different from 0 . Therefore

The characteristic $\mathbf{X}(V, W)$ of two polynomials $V(x), W(x)$ with variable coefficients can change only when the resultant $R(V, W)$ vanishes.

The characteristic $\mathrm{X}\left(V, V^{\prime}\right)$ of a polynomial $V(x)$ with variable coefficients, and therefore the number of the zero intervals of $V(x)$, can change only when the discriminant $D(V)$ vanishes.

7. From the equations (4) and (6) of $\S 5$ it follows that

$$
\text { (1) } f(x)-S(x) / r^{\prime}=\left(x-\xi_{1}\right)\left(x-\xi_{2}\right) \cdots\left(x-\xi_{\nu}^{\top}\right) Q(x) \text {. }
$$

We have therefore shown for any polynomial $f(x)=\sum_{i=0}^{n} a_{i} x^{n-i}$ which has rational coefficients and $\nu$ zero intervals that by a change in the coefficients $a_{n-\nu+1}, \cdots, a_{n}$ which is as small as we please the polynomial becomes resolvable into a product of $\nu$ linear factors and a factor $Q(x)$ which has no zero intervals, all these factors having rational coefficients. For the case in which $\nu=n$, the proof of Kronecker's theorem is now complete. But for the case in which $\nu<n$, it remains to prove that, by another variation of the coefficients as small as we please, $Q(x)$ becomes resolvable into a product of quadratic factors with rational coefficients.

8. Let $Q(x)=a_{0} F(x)$ and $n-\nu=m$. Then $F(x)$ may be written in the form

$$
F(x)=x^{m}-c_{1} x^{m-1}+\cdots+(-1)^{m} c_{m} .
$$

This function has rational coefficients, no zero intervals, and its discriminant $D$ is not 0 . 
Form the function

$$
\begin{aligned}
F_{1}(x)=\left(x-x_{1}\right)\left(x-x_{2}\right) \cdots\left(x-x_{m}\right) & \\
& =x^{m}-f_{1} x^{m-1}+\cdots+(-1)^{m} f_{m},
\end{aligned}
$$

where $x_{1}, x_{2}, \cdots, x_{m}$ are independent variables.

Select any two of these variables, say $x_{1}$ and $x_{2}$, and form the function

$$
g=p\left(x_{1}+x_{2}\right)+x_{1} x_{2}
$$

where $p$ represents a rational integer.

Of the $m$ ! permutations of $x_{1}, x_{2}, \cdots, x_{m}$ there are $2(m-2)$ ! which leave $g$ interchanged. But if we apply all the $m !$ permutations we obtain a system of $\rho=m(m-1) / 2$ conjugate functions

$$
g_{1}, g_{2}, \cdots, g_{\rho}
$$

of which one, say $g_{1}$, is $g$ itself. Moreover since the difference of any two of these functions, namely

$$
p\left(x_{h}+x_{k}\right)+x_{h} x_{k}-p\left(x_{i}+x_{j}\right)-x_{i} x_{j},
$$

cannot vanish for all values of $p$ unless $x_{h}=x_{i}, x_{k}=x_{j}$ or $x_{h}=x_{j}, x_{k}=x_{i}$, we can always so determine $p$ (and we shall suppose this to have been done) that the functions $g_{1}, g_{2}, \cdots, g_{\rho}$ are all different, provided, of course, that the discriminant of $F_{1}(x)$ is not 0 . The function $g=g_{1}$ is therefore a root of an equation of the $\rho$ th degree

$$
\prod_{i=1}^{\rho}\left(z-g_{i}\right)=\phi\left(z, f_{1}, f_{2}, \cdots, f_{m}\right)=0
$$

with coefficients which are rational integral functions of the $f_{i}$ 's. The discriminant of this equation is not 0 and the equation is irreducible in the domain of rationality of the $f_{i}$ 's.

Every rational integral symmetric function of the variables $x_{1}, x_{2}$, is a rational integral function of $g$, of degree $\rho-1$ at most, with coefficients which are rational in $f_{1}, f_{2}, \cdots, f_{m}$.

For let $\gamma$ be such a function and let $\gamma_{1}, \gamma_{2}, \cdots, \gamma_{\rho}$, where $\gamma_{1}=\gamma$, be its conjugate functions arranged to correspond to the conjugate functions $g_{1}, g_{2}, \cdots, g_{\rho}$. By Lagrange's interpolation formula, the function $f(z)$ whose values for $z=g_{1}$, $g_{2}, \cdots, g_{\rho}$ are $\gamma_{1}, \gamma_{2}, \cdots, \gamma_{\rho}$ respectively is

(6) $f(z)=\sum_{i=1}^{\rho} \frac{\phi(z)}{z-g_{i}} \frac{\gamma_{i}}{\phi^{\prime}\left(g_{i}\right)}=\frac{s_{0} z^{\rho-1}+s_{1} z^{\rho-2}+\cdots+s_{\rho-1}}{\phi^{\prime}\left(g_{1}\right) \phi^{\prime}\left(g_{2}\right) \cdots \phi^{\prime}\left(g_{\rho}\right)}$, 
where the coefficients $s_{0}, s_{1}, \cdots, s_{\rho-1}$ are rational integral functions of $f_{1}, \cdots, f_{m}$ and the denominator is the discriminant of $\phi(z)=0$. Therefore

$$
\gamma=f(g)=\frac{s_{0} g^{\rho-1}+s_{1} g^{\rho-2}+\cdots+s_{\rho-1}}{\operatorname{Disc} \phi(z)} .
$$

The function $F_{1}(x)$ can be reduced to the form

$$
F_{1}(x)=\left[x^{2}-\left(x_{1}+x_{2}\right) x+x_{1} x_{2}\right] Q\left(x, x_{1}, x_{2}, f_{1}, \cdots, f_{m}\right),
$$

where $Q$ is symmetric with respect to $x_{1}, x_{2}$, and therefore, by the theorem just demonstrated, to the form

$$
F_{1}(x)=\left[x^{2}-\varphi(g) x+\psi(g)\right] Q\left(x, g, f_{1}, \cdots, f_{m}\right),
$$

where $\varphi(g), \psi(g)$ and $Q$ are rational with respect to the $f_{i}$ 's and $g$ and integral with respect to $g$. But from (8) the congruence $F_{1}(x)-\left[x^{2}-\varphi(z) x+\psi(z)\right] Q\left[x, z, f_{1}, \cdots, f_{m}\right] \equiv 0(\bmod z-g)$ follows, and from this in turn, since $\phi\left(z, f_{1}, \cdots, f_{m}\right)$ is the irreducible function in the domain of rationality of the $f_{i}$ 's of which $z-g$ is a factor, the congruence

$$
\begin{array}{r}
F_{1}(x)-\left[x^{2}-\varphi(z) x+\psi(z)\right] Q\left(x, z, f_{1}, \cdots, f_{m}\right) \equiv 0 \\
{\left[\bmod \phi\left(z, f_{1}, \cdots, f_{m}\right] .\right.}
\end{array}
$$

If in (9), we substitute for the $f_{i}$ 's the corrresponding coefficients of the given function $F(x)$, we obtain

$$
\begin{array}{r}
F(x)-\left[x^{2}-\varphi(z) x+\psi(z)\right] Q\left[x, z, c_{1}, \cdots, c_{m}\right] \equiv 0 \\
{\left[\bmod \phi\left(z, c_{1}, \cdots, c_{m}\right)\right],}
\end{array}
$$

where the coefficients of $\varphi(z)$ and $\psi(z)$ are now rational functions of the $c_{i}$ 's. But from (10) it follows that if we carry out the complete division of $F(x)$ by $x^{2}-\varphi(z) x+\psi(z)$ we shall obtain a remainder which can be reduced to the form $\left[r_{0}(z) x+r_{1}(z)\right] \phi(z)$, where $r_{0}(z), r_{1}(z)$ are integral functions of $z$, and $\phi(z)$ stands for $\phi\left(z, c_{1}, \cdots, c_{m}\right)$. Hence the result of this division may be written

$$
\begin{array}{r}
F(x)-\left[x^{2}-\varphi(z) x+\psi(z)\right] Q_{1}\left(x, z, c_{1}, \cdots, c_{m}\right) \\
=\left[r_{0}(z) x+r_{1}(z)\right] \phi(z) .
\end{array}
$$

Since by hypothesis Disc $F(x)$ is different from 0 , so also is Disc $\phi(z)$. Therefore $\phi(z)$ has no multiple factors. We proceed to show that $\phi(z)$ has $\mu=m / 2$ zero intervals. 
With the given function $F(x)$, which has no zero intervals, associate the function $\bar{F}(x)=F(x)+\tau F^{\prime}(x)$, where $\tau$ is a constant so chosen that Disc $\left[F(x)+t F^{\prime}(x)\right]$ does not vanish as $t$ varies from 0 to $\tau$, and therefore $\bar{F}(x)$, like $F(x)$, has no zero intervals. Then $\mathrm{X}(F, F)=0(\S 6)$.

Again let $f(x)=\Pi\left[x^{2}-2 a_{i} x+\left(a_{i}{ }^{2}+b_{i}{ }^{2}\right)\right]$, where the $a_{i}$, $b_{i}$ are any pairs of rational integers, all different; also let $\bar{f}(x)=f(x)+\tau f^{\prime}(x)$ be a polynomial related to $f(x)$ as is $\bar{F}(x)$ to $F(x)$. Then $\mathrm{X}(f, \bar{f})=0$.

Finally form the pair of functions

$$
\begin{aligned}
& F_{t}(x)=(1-t) f(x)+t F(x), \\
& \bar{F}_{\iota}(x)=(1-t) \bar{f}(x)+t \bar{F}(x), \quad(0 \leqq t \leqq 1),
\end{aligned}
$$

Evidently $F_{t}(x)$ and $\bar{F}_{t}(x)$, as thus defined, have positive values only. Therefore, if they have a common factor for any value of $t$ in the interval $(0,1)$, this factor must be of even degree, hence of the second degree at least. But if $F_{t}(x)$ and $\bar{F}_{t}(x)$ are to have a common factor of the second degree, $t$ must satisfy not only the condition $R\left(F_{t}, \bar{F}_{t}\right)=0$ but also the condition $\partial R\left(F_{t}, \bar{F}_{t}\right) / \partial a=0$, where $a$ denotes the term in either $F_{t}(x)$ or $\bar{F}_{\iota}(x)$ which does not involve $x ;^{*}$ and therefore the coefficients of $F(x)$ and $f(x)$ must satisfy the condition $R\left[R\left(F_{t}, \bar{F}\right), \partial R\left(\overline{F_{t}}, F_{t}\right) / \partial a\right]=0$. This last condition is not an identity. Hence the numbers $a_{i}, b_{i}$ on which the coefficients

* It may be proved as follows that if the functions $V(x)=v_{0}+v_{1} x$ $+\cdots+v_{m} x^{m}$ and $W(x)=w_{0}+w_{1} x+\cdots+w_{n} x^{n}$ are to have a common factor of the second degree, to the condition $R=R(V, W)=0$ there must be added the condition $\partial R / \partial v_{0} \equiv 0$. For the functions $V(x)-V(u)$ and $W(x)-W(u)$ have the common factor $x-u$, and therefore

$$
R\left(v_{0}-V(u), v_{1}, \cdots, v_{m}, w_{0}-W(u), w_{1}, \cdots, w_{n}\right)=0
$$

identically. But if we take the derivative of this identity with respect to $v_{1}$, develop the result in powers of $V(u)$ and $W(u)$, and then replace $u$ by $x$, we obtain an identity which is equivalent to the congruence

$$
\left.\frac{\partial R}{\partial v_{0}} x+\frac{\partial R}{\partial v_{1}} \equiv 0 \quad \text { (modd. } V(x), W(x)\right)
$$

Hence if $V(x)$ and $W(x)$ have a common factor of the second degree both $\partial R / \partial v_{0}=0$ and $\partial R / \partial v_{1}=0$. But $\partial R / \partial v_{1}=0$ is not an independent condition; for since $R=0$ is a sufficient as well as a necessary condition for the existence of a common factor of at least the first degree, if both $R=0$ and $\partial R / \partial v_{0}=0$ it follows from (1) that $\partial R / \partial v_{1}=0$. 
of $f(x)$ depend may be so chosen that the condition is not satisfied. Suppose such a choice to have been made. We then have $R\left(F_{t}, \bar{F}_{t}\right) \neq 0$, and therefore Disc $F_{t}(x) \neq 0$, for all values of $t$ in the interval $(0,1)$.

Let $\phi_{0}(z)$ be the polynomial obtained from $\phi\left(z, f_{i}\right)$ by replacing each $(-1)^{i} f_{i}$ by the corresponding coefficient of $f(x)$ expressed as a polynomial in $x$. The equation $\phi_{0}(z)=0$ has $\mu$ real roots, namely, $2 p a_{i}+\left(a_{i}{ }^{2}+b_{i}{ }^{2}\right)(i=1,2, \cdots, \mu)$. Hence $X\left(\phi, \phi^{\prime}\right)=\mu / 2$.

As $t$ varies from 0 to 1 , and therefore $F_{t}(x)$ from $f(x)$ to $F(x)$, the function

$$
\phi_{t}(z)=(1-t) \phi_{0}(z)+t \phi(z)
$$

varies from $\phi_{0}(z)$ to $\phi(z)$. Since Disc $F_{t}(x)$ never vanishes during this variation, the like is true of Disc $\phi_{t}(z)$. Therefore since $\mathrm{X}\left(\phi_{l}, \phi_{t}{ }^{\prime}\right)$ is $\mu / 2$ when $t=0$ it is also $\mu / 2$ when $t=1$. In other words, $\phi(z)$ has $\mu=m / 2$ zero intervals, as was to be proved.

Therefore, if $s$ denote an integer (determined as in $\S 4$ ) such that no $z$-interval of the magnitude $1 / s$ contains zero intervals of both $\phi(z)$ and $\phi^{\prime}(z)$, and if $D=$ Disc $\phi(z)$, we can, as in $\S 5$, find $\mu$ intervals

$$
\left(\zeta_{1}{ }^{\prime}, \zeta_{1}{ }^{\prime \prime}\right),\left(\zeta_{2}{ }^{\prime}, \zeta_{2}{ }^{\prime \prime}\right), \cdots,\left(\zeta_{\mu}{ }^{\prime}, \zeta_{\mu}{ }^{\prime \prime}\right)
$$

of the magnitude $1 / r s|D|$ such that

$$
\begin{aligned}
& \phi\left(\zeta_{i}{ }^{\prime}\right) \phi\left(\zeta_{i}{ }^{\prime \prime}\right)<0 \text { and }|\phi(z)|<1 / r \text { for } \zeta_{i}{ }^{\prime} \leqq z \leqq \zeta_{i}{ }^{\prime \prime} \\
& (i=1,2, \cdots, \mu) \text {, }
\end{aligned}
$$

where $r$ is an integer which may be taken as great as we please.

If $\zeta_{i}$ denote any number in $\left(\zeta_{i}^{\prime}, \zeta_{i}{ }^{\prime \prime}\right)$ and we set $z=\zeta_{i}$ in (11), we have

$$
\begin{array}{r}
F(x)-\left[x^{2}-\varphi\left(\zeta_{i}\right) x+\psi\left(\zeta_{i}\right)\right] Q\left(x, \zeta_{i}, c_{1}, \cdots, c_{m}\right) \\
=\left[r_{0}\left(\zeta_{i}\right) x+r_{1}\left(\zeta_{i}\right)\right] \phi\left(\zeta_{i}\right),
\end{array}
$$

where the right member is a linear function of $x$ with rational coefficients which are numerically less than $r_{0}\left(\zeta_{i}\right) / r$ and $r_{1}\left(\zeta_{i}\right) / r$ respectively and can therefore be made as small as we please by a suitable choice of $r$. Moreover, as in $\S 5$, we can determine an integer $\bar{r}$ such that for $r>\bar{r}$ each of the products $\left[x^{2}-\varphi\left(\zeta_{i}\right) x+\psi\left(\zeta_{i}\right)\right] Q\left(x, \xi_{i}, c_{1}, \cdots, c_{m}\right)(i=1,2, \cdots, \mu)$ is, like $F(x)$, without zero intervals. 
It has therefore been shown that we can find $\mu$ quadratic factors with rational coefficients and without zero intervals

$$
\begin{array}{r}
G_{1}=x^{2}-\phi\left(\zeta_{1}\right) x+\psi\left(\zeta_{1}\right), G_{2}=x^{2}-\psi\left(\zeta_{2}\right) x+\psi\left(\zeta_{2}\right), \cdots, \\
G_{\mu}=x^{2}-\psi\left(\zeta_{\mu}\right) x+\psi\left(\zeta_{\mu}\right)
\end{array}
$$

such that each of the remainders $\operatorname{Rem}\left(F / G_{i}\right)$ will be as small as we please. But from this it follows that the remainder Rem $\left(F / G_{1}, G_{2}, \cdots, G_{\mu}\right)$ can be made as small as we please.

For if no two of the functions $G_{i}$ have a common factor, and we shall show that this is the case, we can find two integral functions $P_{k}, Q_{k}$, such that

$$
P_{k} \cdot G_{1} G_{2} \cdots G_{k-1} G_{k+1} \cdots G_{\mu}+Q_{k} G_{k}=R_{k},
$$

where $P_{k}$ is of the first degree at most, both $P_{k}$ and $Q_{k}$ are integral with respect to the coefficients of the functions $G_{i}$, and $R_{k}$ is the resultant $R\left[G_{k}, G_{1} G_{2} \cdots G_{k-1} G_{k+1} \cdots G_{\mu}\right]$.

Let $P_{k}^{\prime}=P_{k} / R_{k}$, so that $P_{k}^{\prime}$ has fractional coefficients, but such as involve only factors of the form $R\left(G_{k}, G_{1}\right), R\left(G_{k}, G_{2}\right)$, ... in their denominators. It will then follow from (16) that

$$
P_{k}^{\prime} \cdot G_{1} G_{2} \cdots G_{k-1} G_{k+1} \cdots G_{\mu} \equiv 1 \quad\left(\bmod G_{k}\right)
$$

and, therefore, if $G=G_{1} G_{2} \cdots G_{\mu}$, that

$$
\sum_{k=1}^{\mu} P_{k}^{\prime} \cdot G_{1} G_{2} \cdots G_{k-1} G_{k+1} \cdots G_{\mu} \equiv 1 \quad(\bmod G) .
$$

But each term in the first member of this congruence is of the degree $m-1$ at most, while $G$ is of degree $m$. Hence

$$
\sum_{k=1}^{\mu} P_{k}^{\prime} \cdot G_{1} G_{2} \cdots G_{k-1} G_{k+1} \cdots G_{\mu}=1,
$$

from which it follows, by multiplying throughout by $F / G$, that

$$
\frac{F}{G}=\frac{F P_{1}^{\prime}}{G_{1}}+\frac{F P_{2}^{\prime}}{G_{2}}+\cdots+\frac{F P_{\mu}^{\prime}}{G_{\mu}},
$$

and therefore that

$$
\begin{aligned}
& \operatorname{Rem}\left(\frac{F}{G}\right)=G_{2} G_{3} \cdots G_{\mu} \operatorname{Rem}\left(\frac{F P_{1}^{\prime}}{G_{1}}\right)+\cdots \\
&+G_{1} G_{2} \cdots G_{\mu-1} \operatorname{Rem}\left(\frac{F P_{\mu}^{\prime}}{G_{\mu}}\right) .
\end{aligned}
$$


$\operatorname{Rem}\left(F / G_{k}\right)$ is $\left[r_{0}\left(\zeta_{k}\right) x+r_{1}\left(\zeta_{k}\right)\right] \phi\left(\zeta_{k}\right)$. Hence Rem $\left(F P_{k}^{\prime} / G_{k}\right)$ is of the form $\left(a_{k} x+b_{k}\right) \phi\left(\zeta_{k}\right) / R_{k}$ where $a_{k}$ and $b_{k}$ are integral functions of the $\zeta_{i}$ 's. Therefore since each $R_{k}$ is different from 0 and each $\left|\phi\left(\zeta_{k}\right)\right|$ is less than $1 / r, \operatorname{Rem}(F / G)$ is a polynomial in $x$ of degree $m-1$ at most with coefficients which by a suitable choice of $r(>\bar{r})$ and the consequent choice of the numbers $\zeta_{k}$ can be made as small as we please.

9. It remains to prove that the resultant of no two of the functions $G_{i}$, say the functions $G_{1}=x^{2}-\phi\left(\zeta_{1}\right) x+\psi\left(\zeta_{1}\right)$ and $G_{2}=x^{2}-\varphi\left(\zeta_{2}\right) x+\psi\left(\zeta_{2}\right)$ can be 0 , that is, less numerically than every assignable number $\delta$. This resultant may be reduced to the form

$$
\begin{aligned}
16 R\left(G_{1}, G_{2}\right)= & \left(\varphi\left(\zeta_{1}\right)-\varphi\left(\zeta_{2}\right)\right)^{4}+2\left(\varphi\left(\zeta_{1}\right)-\varphi\left(\zeta_{2}\right)\right)^{2} \\
& \times\left[4 \psi\left(\zeta_{1}\right)-\varphi^{2}\left(\zeta_{1}\right)+4 \psi\left(\zeta_{2}\right)-\varphi^{2}\left(\zeta_{2}\right)\right] \\
& +\left[4\left(\psi\left(\zeta_{1}\right)-\psi\left(\zeta_{2}\right)\right)-\left(\varphi^{2}\left(\zeta_{1}\right)-\varphi^{2}\left(\zeta_{2}\right)\right]^{2}\right.
\end{aligned}
$$

Both $4 \psi\left(\zeta_{1}\right)-\varphi^{2}\left(\zeta_{1}\right)$ and $4 \psi\left(\zeta_{2}\right)-\varphi^{2}\left(\zeta_{2}\right)$ are positive. Hence $R\left(G_{1}, G_{2}\right)$ cannot be numerically less than every assignable number unless the same is true of both $\varphi\left(\zeta_{1}\right)-\varphi\left(\zeta_{2}\right)$ and $\psi\left(\zeta_{1}\right)-\psi\left(\zeta_{2}\right)$. But, as we proceed to show, at least one of these differences is numerically greater than a definite positive number.

In defining the function $g=p\left(x_{1}+x_{2}\right)+x_{1} x_{2}$ we subjected the integer $p$ to the single condition implied in the requirement that the discriminant of the equation $\phi\left(z, f_{i}\right)=0$ of which $g$ is a root be not 0 . We shall now define $p$ more closely, as follows. Let

$$
p=2 q+t \text { where } t \geqq q \geqq 1,
$$

determining the integer $q$ also so that the equation $\phi_{1}\left(z, f_{i}\right)=0$ of which the function $g_{1}=q\left(x_{1}+x_{2}\right)+x_{1} x_{2}$ is a root shall not have a zero discriminant. This function $g_{1}$ is a rational integral function of $g$; let $g_{1}=\theta\left(g, f_{i}\right)$. The equations $\phi\left(z, f_{i}\right)=0$ and $\phi_{1}\left[\theta(z), f_{i}\right]=0$, of which the first is irreducible, have the root $g$ in common. Therefore $\phi_{1}\left[\theta(z), f_{i}\right]$ is exactly divisible by $\phi\left(z, f_{i}\right)$. Hence, if in these functions we replace the $f_{i}$ 's by the corresponding coefficients $c_{i}$ of $F(x)$ and call the resulting polynomials $\phi(z)$ and $\phi_{1}[\theta(z)]$, we have

$$
\phi_{1}[\theta(z)]=\phi(z) Q(z),
$$

where $Q(z)$ is integral and all the coefficients are rational in the coefficients of $F(x)$. If, therefore, any interval $\left(z^{\prime}, z^{\prime \prime}\right)$ con- 
tains a zero interval of $\phi(z)$, the interval to which the values which $\theta(z)$ takes in $\left(z^{\prime}, z^{\prime \prime}\right)$ belong will contain a zero interval of $\phi_{1}(z)$.

Let $1 / s_{1}$ represent the magnitude of an interval which cannot contain zero intervals of both $\phi_{1}(z)$ and $\phi_{1}{ }^{\prime}(z)$, the integer $s_{1}$ being determined as in $\S 5$; let $u$ be an integer greater than all the values of $|[\theta(z+\sigma)-\theta(z)] / \sigma|,|\sigma| \leqq 1$, in the interval $\left(-c_{1}, c_{1}\right)$ in which all the zero intervals of $\phi_{1}(z)$ lie; finally, to the conditions already stated for determining $s$ for $\phi(z)$ add $s \geqq s_{1} u$. We shall then have for all values of $z$ in $\left(-c_{1}, c_{1}\right)$

$$
|\theta(z+\sigma)-\theta(z)|<|\sigma| \frac{s}{s_{1}} \quad(|\sigma| \leqq 1) .
$$

Therefore to values of $z$ belonging to intervals of magnitude $1 / s$ and $1 / r s|D|$ respectively there correspond values of $\theta(z)$ belonging to intervals of magnitude $1 / s_{1}$ and $1 / r s_{1}|D|$. The numbers $\zeta_{1}$ and $\zeta_{2}$ belong to zero intervals $\left(\zeta_{1}^{\prime}, \zeta_{1}{ }^{\prime \prime}\right)$ and $\left(\zeta_{2}{ }^{\prime}, \zeta_{2}{ }^{\prime \prime}\right)$ of $\phi(z)$ of magnitude $1 / r s|D|$. Hence $\theta\left(\zeta_{1}\right)$ and $\theta\left(\zeta_{2}\right)$ belong to intervals of magnitude $1 / r s_{1}|D|$ which it was shown above are zero intervals of $\phi_{1}(z)$. But from this and the fact that no interval of the magnitude $1 / s_{1}$ can contain zero intervals of both $\phi_{1}(z)$ and $\phi_{1}{ }^{\prime}(z)$ it follows (see $\S 5$ ) that $\left|\theta\left(\zeta_{1}\right)-\theta\left(\zeta_{2}\right)\right|>1 / s_{1}$. Therefore, if for brevity we set $\theta\left(\zeta_{1}\right)=a_{1}, \theta\left(\zeta_{2}\right)=a_{2}, \zeta_{1}=b_{1}, \zeta_{2}=b_{2}$, we have

Since

$$
\left|a_{1}-a_{2}\right|>\frac{1}{s_{1}} \text { and }\left|b_{1}-b_{2}\right|>\frac{1}{s} \text {. }
$$

$$
\begin{aligned}
p\left(x_{1}+x_{2}\right)+x_{1} x_{2} & =g, \quad q\left(x_{1}+x_{2}\right)+x_{1} x_{2}=\theta(g), \\
x_{1}+x_{2} & =\varphi(g), \quad x_{1} x_{2}=\psi(g)
\end{aligned}
$$

we have, on replacing $g$ by $\zeta_{i}$,

$$
p \varphi\left(\zeta_{i}\right)+\psi\left(\zeta_{i}\right)=\zeta_{i}, \quad q \varphi\left(\zeta_{i}\right)+\psi\left(\zeta_{i}\right)=\theta\left(\zeta_{i}\right)
$$

and therefore

$$
\varphi\left(\zeta_{i}\right)=\frac{\theta\left(\zeta_{i}\right)-\zeta_{i}}{q-p}, \quad \psi\left(\zeta_{i}\right)=\frac{p \theta\left(\zeta_{i}\right)-q \zeta_{i}}{p-q} .
$$

It therefore only remains to prove that one or other of the quantities

$$
d_{1}=a_{1}-a_{2}-\left(b_{1}-b_{2}\right), \quad d_{2}=p\left(a_{1}-a_{2}\right)-q\left(b_{1}-b_{2}\right)
$$


must be numerically greater than some definite positive number. We shall show that $1 / s_{1}$ is such a number.

For if $\left|d_{1}\right|<1 / s_{1}$ then $b_{1}-b_{2}=a_{1}-a_{2} \pm \delta / s_{1}$ where $0 \leqq \delta<1$, and therefore $d_{2}=(p-q)\left(a_{1}-a_{2}\right) \pm \delta q / s_{1}$, which by $(20)$ and (23) is numerically greater than $1 / s_{1}$.

And if $\left|d_{2}\right|<1 / s_{1}$, then $q\left(b_{1}-b_{2}\right)=p\left(a_{1}-a_{2}\right) \pm \delta^{\prime} / s_{1}$, where $0 \leqq \delta^{\prime}<1$ and therefore $d_{1}=-(q+t)\left(a_{1}-a_{2}\right) / q$ $\pm \delta^{\prime} / q s_{1}$, which again is numerically greater than $1 / s_{1}$.

10. Kronecker's theorem has now been demonstrated. For referring to equations (1) of $\S 7$ and (18) of $\S 8$ it will be seen that if we set

$$
F_{r^{\prime} r}(x)=S(x) / r^{\prime}+a_{0}\left(x-\xi_{1}\right)\left(x-\xi_{2}\right) \cdots\left(x-\xi_{\nu}\right) \cdot \operatorname{Rem} F / G
$$

we shall have

$$
\begin{aligned}
f(x)-F_{t^{\prime r}}(x)=a_{0}\left(x-\xi_{1}\right)\left(x-\xi_{2}\right) \cdots\left(x-\xi_{\nu}\right) \\
\quad \times\left(x^{2}-\varphi\left(\zeta_{1}\right) x+\psi\left(\zeta_{1}\right)\right) \cdots\left(x^{2}-\varphi\left(\zeta_{\mu}\right) x+\psi\left(\zeta_{\nu}\right)\right)
\end{aligned}
$$

We have given methods by which $\nu$, the numbers $\xi_{i}$ and $\zeta_{i}$, the functions $\varphi(\zeta), \psi(\zeta)$, and the remainder function $F_{r^{\prime} r}(x)$ may be determined from the coefficients of the given polynomial $f(x)=\sum_{i=0}^{n} a^{i} x^{n-i}$ by a finite number of rational operations. The function $F_{r^{\prime} r}(x)$ is of degree $n-1$ at most. Its coefficients are all numerically less than $k / r^{\prime}$ or $k / r$, where $k$ is a determinable positive number, and can therefore be made as small as we please by assigning sufficiently great values to $r^{\prime}$ and $r$ and then making the corresponding determinations of the numbers $\xi_{i}$ and $\zeta_{i}$. And when the coefficients of $f(x)$ are subjected to the variation which consists in subtracting from them the corresponding coefficients of $F_{r^{\prime} r}(x)$, the resulting polynomial is a product of $\nu$ linear and $(n-\nu) / 2$ quadratic factors, all with rational coefficients.

In conclusion it may be remarked that if recognition be accorded the irrational numbers, and we represent the limits which the numbers $\xi_{i}$ and $\zeta_{i}$ approach as $r^{\prime}$ and $r$ approach infinity by $\xi_{i}{ }^{\prime}$ and $\zeta_{i}{ }^{\prime}$ respectively, it will follow from (1), since $\lim F_{r^{\prime} r}(x)=0$, that

$$
\begin{array}{r}
f(x)=a_{0}\left(x-\xi_{1}{ }^{\prime}\right) \cdots\left(x-\xi_{\nu}{ }^{\prime}\right)\left(x^{2}-\varphi\left(\zeta_{1}{ }^{\prime}\right) x+\psi\left(\zeta_{1}{ }^{\prime}\right)\right) \\
\cdots\left(x^{2}-\varphi\left(\zeta_{\mu}{ }^{\prime}\right) x+\psi\left(\zeta_{\mu}{ }^{\prime}\right)\right)
\end{array}
$$


so that from the result of Kronecker's discussion there follows, for numerical equations, a proof of the fundamental theorem of algebra as ordinarily stated.

PRINCETON UNIVERSITY, December, 1913.

\section{TWO CONVERGENCY PROOFS.}

BY PROFESSOR ARNOLD EMCH.

(Read before the American Mathematical Society, December 30, 1913.)

\section{Introduction.}

IN the study of automorphic functions defined within a fundamental domain $G$ formed by two non-intersecting circles in the "elliptic" case and by two tangent circles in the "trigonometric" case, it is necessary to prove the convergence of certain fundamental series, as has been shown by Schottky recently.* In the first case the substitutions of the cyclic group associated with it may be written in the form

$$
\frac{x_{\lambda}-a}{x_{\lambda}-b}=q^{\lambda} \frac{x-a}{x-b},
$$

where $\lambda$ may assume all integral real values between $-\infty$ and $+\infty$ and where $a$ and $b$ are invariant in all substitutions of the group. When $x$ describes the circle $K_{-1}$ of the fundamental region $G, x_{1}$ describes the circle $K_{1}$ forming the other boundary of $G . \quad x_{\lambda}$ describes a circle $K_{\lambda}$ and all circles $K_{\lambda}$ of the pencil with $a$ and $b$ as limiting points divide the whole $x$-plane into a set of regions corresponding to the substitutions of the group. $\dagger$ Schottky bases his proof of the convergence of the series $\Sigma r_{\lambda}$ of the radii of the circles $K_{\lambda}$ on the invariance of the expression

$$
\frac{\left(r_{\lambda}^{2}+r_{\lambda+1}^{2}-e_{\lambda}^{2}\right)^{2}}{4 r_{\lambda}^{2} r_{\lambda+1}^{2}}-1 \text {. }
$$

* "Ueber die Funktionenklasse, die der Gleichung $F\left(\frac{\alpha x+\beta}{\gamma x+\delta}\right)=F(x)$ genügt," Journal für reine und angewandte Mathematik, vol. 143, pp. 1-24 (May, 1913).

† See also Klein-Fricke, Vorlesungen über die Theorie der elliptischen Modulfunctionen, vol. 1, pp. 163-207. 